\title{
The Relationship between the Changes of Blood NT-proBNP Levels and Postoperative Atrial Fibrillation Occurance in Coronary Artery By-Pass Grafting Surgery Patients
}

\author{
Davut Azboy' ${ }^{1}$ Erdal Simsek ${ }^{2}$, Burak Erdolu3 ${ }^{3}$, Zeki Temizturk ${ }^{1}$, \\ Mehmet Ali Yilmaz ${ }^{4}$, Kasim Karapinar ${ }^{5}$ \\ ${ }^{1}$ Department of Cardiovascular Surgery, Elazig Training and Research Hospital, Elazig, Turkey \\ ${ }^{2}$ Department of Cardiovascular Surgery, Turkiye Yuksek Ihtisas Training and Research Hospital, Ankara, Turkey \\ ${ }^{3}$ Department of Cardiovascular Surgery, Bursa Yuksek Ihtisas Training and Research Hospital, Bursa, Turkey \\ ${ }^{4}$ Department of Cardiovascular Surgery, Tarsus Madicalpark Hospital, Mersin, Turkey \\ ${ }^{5}$ Department of Cardiovascular Surgery, Diskapi Yildirim Beyazit Training and Research Hospital, Ankara, Turkey \\ Email: *ofdogan_md@yahoo.com
}

Received 20 December 2015; accepted 3 January 2016; published 7 January 2016

Copyright (C) 2016 by authors and OALib.

This work is licensed under the Creative Commons Attribution International License (CC BY).

http://creativecommons.org/licenses/by/4.0/

(c) (i) Open Access

\section{Abstract}

Background: The new onset of atrial fibrillation (NOAF) can be occurred after coronary artery bypass grafting (CABG) surgery. NOAF can be occurred because of postoperative severe hemodynamic instability, long duration of ICU and hospital staying time, morbidity and mortality. Our aim of this study was to investigate whether N-terminal pro-brain natriuretic peptide (NT-proBNP) is a cause of NOAF after CABG. Methods: Forty CABG patients were enrolled for this study. Twenty patients operated on cardiopulmonary bypass (Group I; $n: 20$ ). The remaining patients have operated using a beating heart technique (Group II; $n$ : 20). The NT-proBNP levels were calculated preand postoperatively. Results: High rate of NOAF was detected in group I patients $(P<0.05)$. In both groups, the NT-proBNP levels were low with sinus rhythm. The NT-proBNP blood levels in different times (T1, T2, T3, T4) among Group I were found higher than Group II. Conclusions: Our research demonstrated that there was a strongly close relationship between blood NT-proBNP levels' and atrial fibrillation occurrence in CABG patients. According to our results to detect postoperative AF development in early time and its treatment, blood NT-proBNP levels can be calculated in those patients for reducing morbidity and mortality due to AF.

\section{Keywords}

Atrial Fibrillation, Coronary Artery Bypass Surgery, NT-proBNP, Open Heart Surgery

${ }^{*}$ Corresponding author.

How to cite this paper: Azboy, D., Simsek, E., Erdolu, B., Temizturk, Z., Yilmaz, M.A. and Karapinar, K. (2016) The Relationship between the Changes of Blood NT-proBNP Levels and Postoperative Atrial Fibrillation Occurance in Coronary Artery By-Pass Grafting Surgery Patients. Open Access Library Journal, 3: e1818. http://dx.doi.org/10.4236/oalib.1101818 


\section{Introduction}

Atrial fibrillation is a common arrhytmias associated with not only increased morbidity after coronary artery bypass grafting but also increased healthcare costs. Many factors are associated with atrial fibrillation onset after coronary artery bypass grafting. The prevalence of AF is $6 \%$ among 65 years old and over, and $0.5 \%-1 \%$ among general population [1]. Especially in older patients AF as a postoperative complication can occur because of postoperative morbidity and mortality. The incidence of postoperative AF after CABG has been reported as $20 \%-40 \%$ of patients [2] [3]. The patient's pre-existing arrhythmia [4], advanced age [4], preoperative beta-blocker medication [5], hypertension, left ventricule dysfunction, angina pectoris, myocard infarction (MI), and right coronary artery stenosis [5] [6] are accepted the important factors for postoperative AF risk. Multiple mechanisms may be involved in postoperative atrial fibrillation including myocardial enzyme and/or high brain natriuretic peptid levels postoperatively. Previous reports have showed that the risk for postoperative AF was the highest immediately postoperatively and at 48 hours. The risk declined to approximately zero within 18 hours postoperatively. Current research demonstrated that predominant risk factors were older age, longer cross clamp time, and concomitant mitral valve procedure.

Natriuretic peptides are vasoactive hormones secreted from various tissues. There are atrial natriuretic peptide (ANP) secreted from atria, brain natriuretic peptide (BNP) secreted more from ventricles, vascular endothelium central nervous system and C-Type natriuretic peptid secreted from the kidney (CNP). ANP is secreted by the increase in atrium wall tension related to the increase in intravascular volume [7]. In chronic and asymptomatic cardiac insufficiency, ANP level increases. Although BNP is secreted from ventricules fundamentally, a little amount is also secreted from atria [8]. BNP level is the susceptible symptom of ventricule overload [9]. Plasma BNP level increases in congestive heart failure, hypertrophic cardiomyopathy, acute MI, hypoxic myocard, primary pulmonary hypertension, and diastolic dysfunction [10] [11]. Active BNP, proBNP, and N-terminal natriuretic peptide (NT-proBNP) are found in plasma and can be measured with immunoassay tests. BNP enables diuresis and vasodilatation, inhibiting the hypertrophy of vascular smooth muscles and cardiac muscle by renin aldosterone production. It has been shown that natriuretic hormones help determine ventricle dysfunction and predict the complications occuring in the early postoperative period [12] [13]. ANP inhibits the collagen synthesis in fibroblasts and the hypertrophy in myocytes [14].

Therefore, in our study, we searched the relationships between new onset AF (NOAF) and NT-proBNP levels in a small number of CABG patients who underwent on-pump or OPCAB procedures.

\section{Methods}

This study has been done between November 2015 and April 2015. Fortycoronary artery disease (CAD) patients required CABG were included in this study. This study has been approved by local ethics committee. The patients divided into two groups according to CABG techniques (Cardiopulmonary bypass (CPB) use) (Group I; n: 20), and beating heart coronary artery revascularisation (Group II; n: 20). AF occurrence in the patients constituted the primary end-point of the study. The demographycs of the patients has been summarized in Table 1 .

The patients who have left ventricular ejection fraction (LVEF) $<45 \%$, severe heart valve disease, preoperative atrial or ventricular arrhytmia, chronic obstructive lung diseases were exluded from the research. In addition, patients with enlarged left or right atrium, who have used any antiarrhythmic drug were not included in the study. The patients' age more than $70 \mathrm{y}$, or with acute coronary syndrome and cardiac failure, emergency operations have also exluded. Patients who had underwent temporary or permanent pacemakers, who required inotropic agents longer than 24 hours postoperatively, intra aortic balloon pumps use requirement, renal function disorders (creatine level $>2 \mathrm{mg} / \mathrm{dl}$ ) were not included for this study.

\subsection{Surgery}

After midline sternotomy incision, aortic and single venous cannulation was performed abd CPB was instituted. After aortic X-Clamp, myocardial protection was provided using an ante grade and retrograde cold bloodcar- 
Table 1. Demographic data of the patients.

\begin{tabular}{cccc}
\hline & Group I & Group II & P value \\
Age & $60.70 \pm 5.38$ & $59.30 \pm 5.56$ & 0.424 \\
EF (\%) & $50.25 \pm 3.54$ & $49.20 \pm 2.61$ & 0.292 \\
LVESD & $3.18 \pm 0.41$ & $3.08 \pm 0.38$ & 0.404 \\
LVEDD & $4.79 \pm 0.62$ & $4.85 \pm 0.55$ & 0.726 \\
LA diameter & $3.28 \pm 0.50$ & $3.03 \pm 0.52$ & 0.135 \\
RA diameter & $3.56 \pm 0.59$ & $3.54 \pm 0.58$ & 0.893 \\
RV diameter & $2.13 \pm 0.21$ & $2.20 \pm 0.18$ & 0.273 \\
Duration of anesthesia (hour) & $241.85 \pm 40.75$ & $216.40 \pm 26.84$ & 0.026 \\
Number of bypass ( $\leq 2)$ & 5 & 10 & 0.102 \\
Inotropicagents usage & 12 & 9 & 0.342 \\
Hyperlipidemia & 18 & 18 & 1.000 \\
Hypertension & 18 & 13 & 0.661 \\
Diabetesmellitus & & 0.523 \\
\hline
\end{tabular}

LVESD: Left Ventricule End Systolic Diameter, LVEDD: Left Ventricule End Diastolic Diameter, LA: Left Atrium, RA: Right Atrium, RV: Right Ventricule, EF: Ejection fraction.

dioplegia. Hot-Shot blood delivery was applied via antegradely before aortic cross clamp release. Moderate hypothermia $\left(32^{\circ} \mathrm{C}-34^{\circ} \mathrm{C}\right)$ was applied in all patients. In patients who underwent beating heart bypass operations, heparin was administered and activating coagulation time (ACT) was provided.

\subsection{Blood NT-proBNP Levels}

The blood taken from peripheral venous line and kept under +4 centigrade degrees until decomposed with a 5 min. centrifugation in 3500 rotation. The decomposed serums were kept in 80 centigrade deep freeze. NTproBNP levels were measured at four stages; preoperatively (T1), 1st day (T2), 3rd day (T3) and 5th day (T4) after the operation. Measurements have been with ELISA Kit Test system.BNP levels have been determined as $\mathrm{pg} / \mathrm{ml}$. The lowest NT-proBNP level that the test could verify was determined as $0.312 \mathrm{pg} / \mathrm{ml}$. Starting from the first day after the operation, all the patients were given Acetylsalicylic acid and beta blocker drugs however; they weren't given any antiaritmic drugs. Their cardiac rhythms were followed by daily 12 lead ECG.AF was defined as the development of irregular ventricular rhythm or P wave deficiency before QRS complex during ECG. Occurrence of AF once created the primary endpoint.

\subsection{Statistical Analysis}

For both groups, Student's t-Test was used to compare age, NT-proBNP levels, EF, LVESD, LVEDD, LA, RA and RV diameters, anesthesia durations, cross clamp and CPB times in group I over AF factors. Again in comparing theses variables among the groups-AF taken into consideration-Student's t-Test was used. Among the categorical variables that took place in our study: distal anastomosis numbers, inotropic agent support, diabetes mellitus, hypertension, hyperlipidemia analysis, Pearson chi square correlation was benefited from. Fisher's Exact Test and Pearson chi square correlation were practised upon for the relationship between categorical variables and AF. The results' being $\mathrm{P}<0.05$ was evaluated at a level of significance.

\section{Results}

The clinical and operational information of group I and II patients and also group I's cross clamp and CPB durations are shown in Table 1. In both groups; age, EF, Left Ventricule End Systolic Diameter (LVESD), Left Ventricule End Diastolic Diameter (LVEDD), Left Atrium (LA), right atrium and right ventricule diameters, 
anesthesia duration, cross clamp and CPB durations were measured. The effects of these parameters on AF occurrence were evaluated. Among Group I patients, AF occurred ones' ages, LVESD, LVEDD, LA, RA, RV diameters were found significantly higher compared to patients with normal rhythm $(\mathrm{P}<0.05)$. EF value in AF patients was found significantly lower compared to sinus rhythm patients $(\mathrm{P}<0.05)$. There wasn't found any significant difference between the AF observed patients' and normal rhythm patients' ages and anesthesia durations in group II (P > 0.05). However, in AF developed patients in group II, LVESD, LVEDD, LA, RA, and RV diameter averages were noted significantly higher than the patients' with normal rhythm $(\mathrm{P}<0.05)$. EF values were noted significantly lower in AF developed patients than in patients of normal rhythm $(\mathrm{P}<0.05)$. When both groups were compared in terms of AF developed patients, there was no significant difference between age, EF, LVESD, LVEDD, LA, RA, RV diameters and anesthesia durations (P > 0.05). All phases of NT-proBNP levels of AF developed patients among group I and II that were found significantly higher than the levels of patients with normal rhythm $(\mathrm{P}<0.05)$ (Table 2).

While NT-proBNP levels in T1 weren't found any significant difference between two groups' AF developed patients, T2, T3, T4 times were found significantly higher in average of AF developed group I patients $(\mathrm{P}<0.05)$ (Table 3). The relationship of anastomosed distal bypass number and inotropic agent usage with AF could not be determined in either group $(\mathrm{P}>0.05)$.

There wasn't a significant difference, in terms of cross clamp and CPB periods, between AF patients and normal sinus rhythm patients among group I $(\mathrm{P}>0.05)$.

\section{Discussion}

Atrial fibrillation is a common arrhythmia after CABG operations. Many factors are associated with NOAF in CABG patients. AF can be cause postoperative morbidity and mortality especially in older patients. The incidence of postoperative AF in CABG patients has been reported as $20 \%-40 \%$ of patients [2] [3]. The patient's pre-existing arrhythmia [4], advanced age [4], preoperative beta-blocker medication [5], hypertension, left ventricle dysfunction, angina pectoris, MI, right coronary artery stenosis [5] [6] are accepted the main important risk factors for postoperative AF. Multiple mechanisms may be involved in postoperative atrial fibrillation including myocardial enzyme release and/or high brain natriuretic peptid postoperatively. Previous reports have showed that the risk for postoperative AF was highest immediately postoperatively and at 48 hours. The risk declined to approximately zero within 18 hours postoperatively. Current research demonstrated that predominant risk factors were older age, longer cross clamp time, and concomitant mitral valve procedure. A number of risk factors including biochemical changes during the ECC have been researched for new onset of AF; however, there is a limited number of studies the relationships between the NOAF and blood proBNP levels.

Table 2. NT-proBNP values in different times.

\begin{tabular}{lcccccc}
\hline & Group I & Group I with AF & P value & Group II & Group II with AF & P value \\
\hline NT proBNP pg/ml (T1) & 15.56 & 30.28 & 0.014 & 13.14 & 21.35 & 0.003 \\
NT proBNP pg/ml (T2) & 16.11 & 35.34 & 0.008 & 16.84 & 24.92 & 0.002 \\
NT proBNP pg/ml (T3) & 18 & 37.17 & 0.001 & 18.49 & 28 & 0.008 \\
NT proBNP pg/ml (T4) & 22.27 & 40 & 0.007 & 19 & 31.48 & 0.000 \\
\hline
\end{tabular}

AF: Atrial Fibrillation, NT-proBNP: N-terminal natriuretic peptide, T1: pre-operation time, T2:1st day after the operation, T3: 3rd day after the operation, T4: 5 th day after the operation (T4). $\mathrm{P}<0.05$ was evaluated at a level of significance.

Table 3. Blood NT proBNP levels (different times) and AF occurrence in Group I and II.

\begin{tabular}{lccc}
\hline & Group I with AF & Group II with AF & P value \\
\hline NT proBNP (T1) & 30.29 & 21.35 & 0.070 \\
NT proBNP (T2) & 35.34 & 24.92 & 0.027 \\
NT proBNP (T3) & 37.17 & 28 & 0.034 \\
NT proBNP (T4) & 40 & 31.48 & 0.036 \\
\hline
\end{tabular}

NT-proBNP; N-terminal natriuretic peptide; AF: Atrial Fibrillation; T1: pre-operation time, T2: 1st day after the operation, T3: 3rd day after the operation, T4: 5th day after the operation. $\mathrm{P}<0.05$ was evaluated at a level of significance. 
As we know that natriuretic peptides are vasoactive hormones secreted from various tissues. Such as atrium or brain. This biochemical marker also secreted from ventricles, vascular endothelium, central nervous system. Ctype natriuretic peptid secreted from the kidney (CNP). Atrial Natriuretic Peptide is secreted fromthe atrium related to high atrial wall tension and intravascular volume loading [7]. BNP is secreted from the ventricle and is also secreted from atria [8]. But, BNP level is a susceptible for symptom of ventricular overloading [9]. Plasma BNP level increases in congestive heart failure, hypertrophic cardiomyopathy, acute MI, hypoxic myocard, primary pulmonary hypertension, diastolic dysfunction [10] [11]. Active BNP, proBNP, N-terminal natriuretic peptide (NT-proBNP) can be measured with immunoassay tests. BNP is a cause of diuresis and vasodilatation, inhibiting the hypertrophy of vascular smooth and cardiac muscle by renin-aldoster one production. It has been shown that natriuretic hormones help determine ventricle dysfunction and predict the complications occuring in the early postoperative period [12]-[14].

It has been demonstrated that in patients with enlarged left atrium and ventricle, and low EF are the risk factors of postoperative AF occurrence [17]. To provide confusing of postoperative AF causes in our patients, we selected the patients without the risk factors including age, preoperative arrhytmia, valvular disease, cardiomegaly related to any reason, etc. in both groups. Indeed, preoperative echocardiographyc examinations showed that there was no significant difference between left ventricular end-systolic and end-diastolic diameter, bi-atrial and bi-ventricular enlargement in both groups. A multivariate analysis has been shown that advanced age is a strong postoperative AF development predictor. A multy-variate analyses demonstrated that hypertension, ReDo CABG were found to be a significant risk factors for NOAF in previous studies. Also, prolonged aortic cross clamping and longer operation [19], anesthesics, insufficient ventilation [4], cardioplegic arrest are the risk factors of AF. In our study, if patients' cross clamp time and CPB durations increased in On-Pump group, these patients were excluded from the study to provide any confusing of the reason of AF occurrence.

AF incidence has been reported between $20 \%$ to $40 \%$ in wide series [2] [3]. Previous studies exhibited that AF frequency did not change in patients underwent beating heart technique [20]-[22]. However, the authors stated that CPB was a risk factor of NOAF [23] [24].

In our study, postoperative AF development rate was found $25 \%$ of the patients in "On-Pump" group. AF developed in $15 \%$ of OPCAB patients. This was statistically significant. The postoperative AF occurrence was significantly low in terms of statistics, in the patients underwent beating heart bypass technique. In our opinion, proBNP levels can be related this important result.

In congestive heart failure and acute MI, NT-proBNP levels is increased [25]. It has been shown that NT-proBNP levels and myocardial ischemia, creatine kinase-MB and ventricular function are related to NOAF [26].

In the presence of minor perioperative myocardial ischemia, NT-proBNP was brought forward to be a sensitive marker of dysfunction after ischemia [27]. Recently in some studies, it has been put forth that perioperative NT-proBNP levels correlate with complications such as; inotropic agency, intra aortic oballoon pump use, length of hospitalization, postoperative AF and mortality [12] [28].

It has been stated that postoperative high proBNP levels are independent risk factors for postoperative AF occurrence [29]. In a previous study included patients with and without left ventricular dysfunction in CABG patients has been reported that no correlation has been detected between blood proBNP levels and NOAF. This study demonstrated that there was no statistical significancy NOAF after surgery when compared to two groups' BNP level changes in post surgery $24 \mathrm{th}$. However, the same authors stated that 48th h post surgery, there was a significant increment in NOAF in theirs groups who have had high BNP levels [30].

The published studies showed the increment of NT-proBNP levels started at 6th $\mathrm{h}$ and reached its peak point on 4-6th days, post surgery. This clinical situation may continued during weeks [28] [30]. In our study, postoperative NT-proBNP levels increased gradually in both goups. We detected highest level of NT-proBNP after On-Pump surgery was related preoperative NT-proBNP levels especially in older age in both groups. To the best of our knowledge this clinical situation has been detected in our research.

Postoperative changes of NT-proBNP levels increased in On-Pump and OPCAB patients. However, we seen that these increament was more common in On-Pump patients. There was a strong relationship between NTproBNP levels and NOAF in this groups. Also, in our study exhibited that there was a significant correlation in older age patients, number of diseased coronary arteries, cross clamp and CPB duration. Therefore, our study suggested that NT-proBNP may be measured in older CABG patients who underwent CPB. The specifity of NT-proBNP has been reported as $69.7 \%-83.3 \%$. 


\section{Conclusion}

Our research demonstrated a positive correlation between NT-proBNP levels and NOAF. Therefore, we suggested that NT-proBNP values could be used as important risk indexes in terms of postoperative AF occurrence after CABG. Therefore, previous studies indicated the risk factors for NOAF postoperatively; we established that NT-proBNP levels could be used in older patients with high preoperative NT-proBNP level, low EF, atrial and a large atrio-ventricular diameter. NT-proBNP level may be investigated as a routine test prior to open heart surgery, extremely important in terms of decreasing foreseen morbidity and mortality. If we detect high level of NT-proBNP before the CABG operation we can choose beating bypass procedure if it is possible.

\section{Limitations of Study}

Our study includes a small number of coronaryartery disease. Therefore, for definition of importance of NTproBNP levels the study can be designed in a large number of patients. Thus, we continued this research programme in our CABG patients. Rhytm and tension Holter monitoring can be decided in these patients to detect transient atrio-ventricular arrhytmia development.

\section{Acknowledgements}

The authors would like to thanks to Prof. Dr. Omer Faruk Dogan, the chief of cardiovascular surgery, because he helped to prepare and revision processess of our manuscript.

\section{References}

[1] Lloyd-Jones, D.M., Wang, T.J., Leip, E.P., Larson, M.G., Levy, D., Vasan, R.S., et al. (2004) Lifetime Risk for Development of Atriyal Fibrillation. Circulation, 110, 1042-1046.

http://dx.doi.org/10.1161/01.CIR.0000140263.20897.42

[2] Cresswell, L.L., Schuessler, R.B., Rosenblom, M. and Cox, J.L. (1993) Hazards of Postoperative Atrial Arrhythmias. Annals of Thoracic Surgery, 56, 539-549. http://dx.doi.org/10.1016/0003-4975(93)90894-N

[3] Creswell, L.L. (1999) Postoperative Atrial Arrhythmias: Risk Factors and Associated Advers Outcomes. Seminars in Thoracic and Cardiovascular Surgery, 11, 303-307. http://dx.doi.org/10.1016/S1043-0679(99)70073-0

[4] Wazni, O.M., Martin, D.O., Marrouche, N.F., Latif, A.A., Ziada, K., Shaaraoui, M., et al. (2004) Plasma B-Type Natriuretic Peptide Levels Predict Postoperative Atrial Fibrillation in Patients Undergoing Cardiac Surgery. Circulation, 110, 124-127. http://dx.doi.org/10.1161/01.CIR.0000134481.24511.BC

[5] Taylor, A.D., Groen, J.G., Thorn, S.L., Lewis, C.T. and Marshall, A.J. (2002) New Insights into Onset Mechanisms of Atrial Fibrillation and Flutter after Coronary Artery Bypass Graft Surgery. Heart, 88, 499-504. http://dx.doi.org/10.1136/heart.88.5.499

[6] Ommen, S.R., Odell, J.A. and Stanton, M.S. (1997) Atrial Arrhythmias after Cardiothoracic Surgery. New England Journal of Medicine, 336, 1429-1434. http://dx.doi.org/10.1056/NEJM199705153362006

[7] Sudoh, T., Kangawa, K., Minamino, N. and Matsuo, H. (1988) A New Natriuretic Peptide in Porcine Brain. Nature, 332, 78-81. http://dx.doi.org/10.1038/332078a0

[8] Hosoda, K., Nakao, K., Mukoyama, M., Saito, Y., Jougasaki, M., Shirakami, G., et al. (1991) Expression of Brain Natriuretic Peptide Gene in Human Heart. Production in the Ventricle. Hypertension, 17, 1152-1155. http://dx.doi.org/10.1161/01.HYP.17.6.1152

[9] Grantham, J.A., Borgeson, D.D. and Burnett Jr., J.C. (1997) BNP: Pathophysiological and Potential Therapeutic Roles in Acute Congestive Heart Failure. American Journal of Physiology, 272, 1077-1083.

[10] Hasegawa, K., Fujiwara, H., Doyama, K., Miyamae, M., Fujiwara, T., Suga, S., et al. (1993) Ventricular Expression of Brain Natriuretic Peptide in Hypertrophic Cardiomyopathy. Circulation, 88, 372-380. http://dx.doi.org/10.1161/01.CIR.88.2.372

[11] Goetze, J.P., Wang, Y.Z., Rehfeld, J.F., Jørgensen, E. and Kastrup, J. (2004) Coronary Angiography Transiently Increases Plasma Pro B Type Natriuretic Peptide. European Heart Journal, 25, 759-764. http://dx.doi.org/10.1016/j.ehj.2004.02.009

[12] Hutfless, R., Kazanegra, R., Madani, M., Bhalla, M.A., Tulua-Tata, A., Chen, A., et al. (2004) Utility of B-Type Natriuretic Peptide in Predicting Postoperative Complications and Outcomes in Patients Undergoing Heart Surgery. Journal of the American College of Cardiology, 43, 1873-1879. http://dx.doi.org/10.1016/j.jacc.2003.12.048

[13] Levin, E.R., Isackson, P.J. and Hu, R.M. (1991) Endothelin Increases Atrial Natriuretic Peptide Production in Cultured 
Rat Diencephalic Neurons. Endocrinology, 128, 2925-2930. http://dx.doi.org/10.1210/endo-128-6-2925

[14] Bex, F. and Corbin, A. (1985) Atrial Natriuretic Factor Simulates Testosterone Production by Mouse Interstitial Cells. European Journal of Pharmacology, 115, 125-126. http://dx.doi.org/10.1016/0014-2999(85)90595-3

[15] Siebert, J., Anisimowicz, L., Lango, R., Rogowski, J., Pawlaczyk, R., Brzezinski, M., et al. (2001) Atrial Fibrillation after Coronary Artery Surgery Bypass Grafting: Does the Type of Procedure Influence the Early Postoperatiferative Incidence? European Journal Cardio-Thoracic Surgery, 19, 455-459. http://dx.doi.org/10.1016/S1010-7940(01)00621-2

[16] Creswell, L.L., Schuessler, R.B., Rosenbloom, M. and Cox, J.L. (1993) Hazards of Postoperative Atrial Arrhythmias. The Annals of Thoracic Surgery, 56, 539-549. http://dx.doi.org/10.1016/0003-4975(93)90894-N

[17] Leung, J.M., Bellows, W.H. and Schiller, N.B. (2004) Impairment of Left Atrial Function Predicts Post-Operative Atrial Fibrillation after Coronary Artery Bypass Graft Surgery. European Heart Journal, 25, 1836-1844. http://dx.doi.org/10.1016/j.ehj.2004.07.014

[18] Moulton, M.J., Creswell, L.L., Mackey, M.E., Cox, J.L. and Rosenbloom, M. (1996) Reexploration for Bleeding Is a Risk Factor for Adverse Outcomes after Cardiac Operations. The Journal of Thoracic and Cardiovascular Surgery, 111, 1037-1046. http://dx.doi.org/10.1016/S0022-5223(96)70380-X

[19] Almassi, G.H., Schowalter, T., Nicolosi, A.C., Aqqarwal, A., Moritz, T.E., Henderson, W.G., et al. (1997) Atrial Fibrillation after Cardiac Surgery: A Major Morbid Event? Annals of Surgery, 226, 501-513. http://dx.doi.org/10.1097/00000658-199710000-00011

[20] Cohn, W.E., Sirois, C.A. and Johnson, R.G. (1999) Atriyal Fibrillation after Minimally Invasive Coronary Artery Bypass Grafting: A Retrospective, Matched Study. The Journal of Thoracic and Cardiovascular Surgery, 117, 298301. http://dx.doi.org/10.1016/S0022-5223(99)70426-5

[21] Staatvedt, K., Fiane, A.E., Sellevold, O. and Nordstrand, K. (1999) Is Atrial Fibrillation Caused by Extracorporeal Circulation? The Annals of Thoracic Surgery, 68, 931-933. http://dx.doi.org/10.1016/S0003-4975(99)00686-4

[22] Salamon, T., Michler, R.E., Knott, K.M. and Brown, D.A. (2003) Off-Pump Coronary Artery Bypass Grafting Does Not Decrease the Incidence of Atrial Fibrillation. The Annals of Thoracic Surgery, 75, 505-507. http://dx.doi.org/10.1016/S0003-4975(02)04305-9

[23] Stamou, S.C., Dangas, G., Hill, P.C., Pfister, A.J., Dullum, M.K., Boyce, S.W., et al. (2000) Atrial Fibrillation after Beating Heart Surgery. American Journal of Cardiology, 86, 64-67. http://dx.doi.org/10.1016/S0002-9149(00)00829-8

[24] Kilger, E., Weis, F.C., Goetz, A.E., Frey, L., Kesel, K., Schütz, A., et al. (2001) Intensive Care after Minimally Invasive and Conventional Coronary Surgery: A Prospective Comparison. Intensive Care Medicine, 27, 534-539. http://dx.doi.org/10.1007/s001340000788

[25] Provenchère, S., Berroeta, C., Reynaud, C., Baron, G., Poirier, I., Desmonts, J.M., et al. (2006) Plasma Brain Natriuretic Peptide and Cardiac Troponin I Concentrations after Adult Cardiac Surgery: Association with Postoperative Cardiac Dysfunction and 1-Year Mortality. Critical Care Medicine, 34, 995-1000. http://dx.doi.org/10.1097/01.CCM.0000206110.94385.C4

[26] Chello, M., Mastroroberto, P., Perticone, F., Cirillo, F., Bevacqua, E., Olivito, S., et al. (2001) Plasma Levels of Atrial and Brain Natriuretic Peptides as Indicators of Recovery of Left Ventricular Systolic Function after Coronary Artery Bypass. European Journal Cardio-Thoracic Surgery, 20, 140-146. http://dx.doi.org/10.1016/S1010-7940(01)00754-0

[27] Cosqrave, J., Foley, J.B., McGovern, E., Bennett, K., Young, V., Tolan, M., et al. (2006) Brain Natriuretic Peptide Elevation and the Development of Atrial Fibrillation Following Coronary Artery Bypass Surgery. Interactive CardioVasc Thoracic Surgery, 5, 111-114. http://dx.doi.org/10.1510/icvts.2005.118265

[28] Hakala, T., Hedman, A., Turpeinen, A., Kettunen, R., Vuolteenaho, O. and Hippeläinen, M. (2002) Prediction of Atrial Fibrillation after Coronary Artery Bypass Grafting by Measuring Atrial Peptide Levels and Preoperative Atrial Dimensions. European Journal Cardio-Thoracic Surgery, 22, 939-943. http://dx.doi.org/10.1016/S1010-7940(02)00565-1

[29] Stockand, J.D. and Sansom, S.C. (1997) Regulation of Filtration Rate by Glomerular Mesangial Cells in Health and Diabetic Renal Disease. American Journal of Kidney Diseases, 29, 971-981. http://dx.doi.org/10.1016/S0272-6386(97)90476-5

[30] Guerin, V., Ayed, S.B., Varnous, S., Golmard, J.L., Leprince, P. and Beaudeux, J.L. (2006) Release of Brain Natriuretic-Related Peptides (BNP, NT-proBNP) and Cardiac Troponins (cTnT, cTnI) in On-Pump and Off-Pump Coronary Artery Bypass Surgery. Surgery Today, 36, 783-789. http://dx.doi.org/10.1007/s00595-006-3247-0 


\section{Abbreviations}

NOAF: new onset of atrial fibrillation

CABG: coronary artery bypass grafting

NT-proBNP: N-terminal pro-brain natriuretic peptide

LVEF: left ventricular ejection fraction

EF: ejection fraction

LVESD: left ventricular end-systolic diameter

LVEDD: left ventricular end-diastolic diameter

LA: left atrium

$\mathrm{RA}$ : right atrium

$\mathrm{RV}$ : right ventricule

CPB: cardiopulmonary by-pass

CNP: C-type natriuretic peptid

MI: myocardial infarctus

OPCAB: off-pump coronary artery bypass

CPB: cardiopulmonary by-pass 